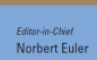

Norbert Euler

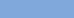

\section{Journal of Nonlinear Mathematical}

\section{Physics}

ISSN (Online): 1402-9251 ISSN (Print): 1776-0852

Journal Home Page: https://www.atlantis-press.com/journals/jnmp

\title{
The heptagon-wheel cocycle in the Kontsevich graph complex
}

Ricardo Buring, Arthemy V. Kiselev, Nina J. Rutten

To cite this article: Ricardo Buring, Arthemy V. Kiselev, Nina J. Rutten (2017) The heptagon-wheel cocycle in the Kontsevich graph complex, Journal of Nonlinear Mathematical Physics 24: Supplement 1, 157-173, DOI:

https://doi.org/10.1080/14029251.2017.1418060

To link to this article: https://doi.org/10.1080/14029251.2017.1418060

Published online: 04 January 2021 


\title{
The heptagon-wheel cocycle in the Kontsevich graph complex
}

\author{
Ricardo Buring \\ Institut für Mathematik, Johannes Gutenberg-Universität, Staudingerweg 9, D-55128 Mainz, Germany \\ rburing@uni-mainz.de \\ Arthemy V. Kiselev \\ Johann Bernoulli Institute for Mathematics and Computer Science, University of Groningen, \\ P.O. Box 407, 9700 AK Groningen, The Netherlands \\ A.V.Kiselev@rug.nl \\ Nina J. Rutten \\ Johann Bernoulli Institute for Mathematics and Computer Science, University of Groningen, \\ P.O. Box 407, 9700 AK Groningen, The Netherlands
}

Received 2 October 2017

Accepted 24 November 2017

\begin{abstract}
The real vector space of non-oriented graphs is known to carry a differential graded Lie algebra structure. Cocycles in the Kontsevich graph complex, expressed using formal sums of graphs on $n$ vertices and $2 n-$ 2 edges, induce - under the orientation mapping - infinitesimal symmetries of classical Poisson structures on arbitrary finite-dimensional affine real manifolds. Willwacher has stated the existence of a nontrivial cocycle that contains the $(2 \ell+1)$-wheel graph with a nonzero coefficient at every $\ell \in \mathbb{N}$. We present detailed calculations of the differential of graphs; for the tetrahedron and pentagon-wheel cocycles, consisting at $\ell=1$ and $\ell=2$ of one and two graphs respectively, the cocycle condition $\mathrm{d}(\gamma)=0$ is verified by hand. For the next, heptagonwheel cocycle (known to exist at $\ell=3$ ), we provide an explicit representative: it consists of 46 graphs on 8 vertices and 14 edges.
\end{abstract}

Keywords: Non-oriented graph complex; differential; cocycle; symmetry; Poisson geometry.

2010 Mathematics Subject Classification: 13D10, 32G81, 53D17, 81S10, also 53D55, 58J10, 90C35

\section{Introduction}

The structure of differential graded Lie algebra on the space of non-oriented graphs, as well as the cohomology groups of the graph complex, were introduced by Kontsevich in the context of mirror symmetry $[10,11]$. It can be shown that by orienting a graph cocycle on $n$ vertices and $2 n-2$ edges (and by adding to every graph in that cocycle two new edges going to two sink vertices) in all such ways that each of the $n$ old vertices is a tail of exactly two arrows, and by placing a copy of a given Poisson bracket $\mathscr{P}$ in every such vertex, one obtains an infinitesimal symmetry of the space of Poisson structures. This construction is universal with respect to all finite-dimensional affine real manifolds (see [12] and [2]). ${ }^{a}$ Until recently two such differential-polynomial symmetry

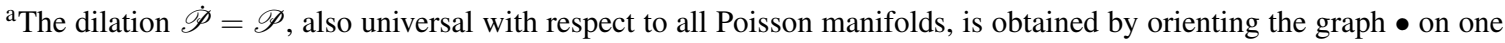
vertex and no edges, yet that graph is not a cocycle, $\mathrm{d}(\bullet)=-\bullet \bullet \neq 0$. The single-edge graph $\bullet \bullet \in$ kerd on two vertices is a cocycle but its bi-grading differs from $(n, 2 n-2)$. However, by satisfying the zero-curvature equation $\mathrm{d}(\bullet \bullet)+$ $\frac{1}{2}[\bullet \bullet, \bullet \bullet]=0$ the graph $\bullet \bullet$ is a Maurer-Cartan element in the graph complex. 
flows were known (of nonlinearity degrees 4 and 6 respectively). Namely, the tetrahedral graph flow $\dot{\mathscr{P}}=\mathscr{Q}_{1: \frac{6}{2}}(\mathscr{P})$ was proposed in the seminal paper [12] (see also [2,3]). Consisting of 91 oriented bivector graphs on $5+1=6$ vertices, the Kontsevich-Willwacher pentagon-wheel flow will presently be described in [7].

The cohomology of the graph complex in degree 0 is known to be isomorphic to the Grothendieck-Teichmüller Lie algebra grt (see [9] and [16]); under the isomorphism, the grt generators correspond to nontrivial cocycles. Using this correspondence, Willwacher gave in [16, Proposition 9.1] the existence proof for an infinite sequence of the Deligne-Drinfel'd nontrivial cocycles on $n$ vertices and $2 n-2$ edges. (Formulas which describe these cocycles in terms of the grt Lie algebra generators are given in the preprint [15].) To be specific, at each $\ell \in \mathbb{N}$ every cocycle from that sequence contains the $(2 \ell+1)$-wheel with nonzero coefficient (e.g., the tetrahedron alone making the cocycle $\gamma_{3}$ at $\ell=1$ ), and possibly other graphs on $2 \ell+2$ vertices and $4 \ell+2$ edges. For instance, at $\ell=2$ the pentagon-wheel cocycle $\gamma_{5}$ consists of two graphs, see Fig. 1 on p. 162 below.

In this paper we describe the next one, the heptagon-wheel cocycle $\boldsymbol{\gamma}_{7}$ from that sequence of solutions to the equation

$$
\mathrm{d}\left(\sum_{\{\text {graphs }\}}(\text { coefficient } \in \mathbb{R}) \cdot(\text { graph with an ordering of its edge set })\right)=0 .
$$

Our representative of the cocycle $\boldsymbol{\gamma}_{7}$ consists of 46 connected graphs on 8 vertices and 14 edges. (This number of nonzero coefficients can be increased by adding a coboundary.) This solution has been obtained straightforwardly, that is, by solving the graph equation $\mathrm{d}\left(\boldsymbol{\gamma}_{7}\right)=0$ directly. One could try reconstructing the cocycle $\boldsymbol{\gamma}_{7}$ from a set of the grt Lie algebra generators, which are known in low degrees. Still an explicit verification that $\boldsymbol{\gamma}_{7} \in$ kerd would be appropriate for that way of reasoning.

In this paper we also confirm that the three cocycles known so far - namely the tetrahedron and pentagon- and heptagon-wheel solutions - span the space of nontrivial cohomology classes which are built of connected graphs on $n \leqslant 8$ vertices and $2 n-2$ edges. At $n=9$, there is a unique nontrivial cohomology class with graphs on nine vertices and sixteen edges: namely, the Lie bracket $\left[\boldsymbol{\gamma}_{3}, \boldsymbol{\gamma}_{5}\right]$ of the previously found cocycles. (Brown showed in [4] that the elements $\sigma_{2 \ell+1}$ in the Lie algebra grt which - under the Willwacher isomorphism - correspond to the wheel cocycles $\gamma_{2 \ell+1}$ generate a free Lie algebra; hence it was expected that the cocycle $\left[\boldsymbol{\gamma}_{3}, \boldsymbol{\gamma}_{5}\right]$ is non-trivial.) To verify that the list of currently known d-cocycles is exhaustive - under all the assumptions which were made about the graphs at our disposal - at every $n \leqslant 9$ we count the dimension of the space of cocycles minus the dimension of the space of respective coboundaries. ${ }^{b}$ Our findings fully match the dimensions from [14, Table 1].

This text is structured as follows. Necessary definitions and some notation from the graph complex theory are recalled in $\S 2$. These notions are illustrated in $\S 3$ where a step-by-step calculation of the (vanishing) differentials $\mathrm{d}\left(\boldsymbol{\gamma}_{3}\right)$ and $\mathrm{d}\left(\boldsymbol{\gamma}_{5}\right)$ is explained. Our main result is Theorem 4 with the heptagon-wheel solution of the equation $\mathrm{d}\left(\boldsymbol{\gamma}_{7}\right)=0$. Also in $\S 4$, in Proposition 5 we verify the count of number of cocycles modulo coboundaries which are formed by all connected graphs on $n$ vertices and $2 n-2$ edges (here $4 \leqslant n \leqslant 9$ ). The graphs which constitute $\boldsymbol{\gamma}_{7}$ are drawn on pp. 169-172 in Appendix A. The code in SAGE programming language, allowing one to calculate the differential

${ }^{\mathrm{b}}$ The proof scheme is computer-assisted (cf. [2,6]); it can be applied to the study of other cocycles: either on higher number of vertices or built at arbitrary $n \geqslant 2$ from not necessarily connected graphs. 
for a given graph $\gamma$ and ordering $\mathrm{E}(\gamma)$ on the set of its edges, is contained in Appendix B; the same code can be run to calculate the dimension of graph cohomology groups.

The main purpose of this paper is to provide a pedagogical introduction into the subject. ${ }^{\mathrm{c}}$ Besides, the formulas of the three cocycle representatives will be helpful in the future search of an easy recipe to calculate all the wheel cocycles $\boldsymbol{\gamma}_{2 \ell+1}$. (No general recipe is known yet, except for a longer reconstruction of those cohomology group elements from the generators of Lie algebra $\mathfrak{g r t}$.) Thirdly, our present knowledge of both the cocycles $\gamma_{i}$ and the respective flows $\mathscr{P}=\mathscr{Q}_{i}(\mathscr{P})$ on the spaces of Poisson structures will be important for testing and verifying explicit formulas of the orientation mapping $\mathrm{O} \vec{r}$ such that $\mathscr{Q}_{i}=\mathrm{O} \vec{r}\left(\boldsymbol{\gamma}_{i}\right)$.

\section{The non-oriented graph complex}

We work with the real vector space generated by finite non-oriented graphs ${ }^{\mathrm{d}}$ without multiple edges nor tadpoles and endowed with a wedge ordering of edges: by definition, an edge swap $e_{i} \wedge e_{j}=$ $-e_{j} \wedge e_{i}$ implies the change of sign in front of the graph at hand. Topologically equal graphs are equal as vector space elements if their edge orderings $E$ differ by an even permutation; otherwise, the graphs are opposite to each other (i.e. they differ by the factor -1 ).

Definition 1. A graph which equals minus itself - under a symmetry that induces a parity-odd permutation of edges - is called a zero graph. In particular (view $\bullet \bullet \bullet$ ), every graph possessing a symmetry which swaps an odd number of edge pairs is a zero graph.

Notation 1. For a given labelling of vertices in a graph, we denote by $i j$ (equivalently, by $j i$ ) the edge connecting the vertices $i$ and $j$. For instance, both 12 and 21 is the notation for the edge between the vertices 1 and 2. (No multiple edges are allowed, hence 12 is the edge. Indeed, by Definition 1 all graphs with multiple edges would be zero graphs.) We also denote by $N(v)$ the valency of a vertex $v$.

Example 2.1. The 4-wheel $12 \wedge 13 \wedge 14 \wedge 15 \wedge 23 \wedge 25 \wedge 34 \wedge 45=I \wedge \cdots \wedge V I I I$ or likewise, the $2 \ell$ wheel at any $\ell>1$ is a zero graph; here, the reflection symmetry is $I \rightleftarrows I I I, V \rightleftarrows V I I$, and $V I \rightleftarrows V I I I$.

Note that every term in a sum of non-oriented graphs $\gamma$ with real coefficients is fully encoded by an ordering $\mathrm{E}$ on the set of adjacency relations for its vertices $v$ (if $N(v)>0$ ). From now on, we assume $N(v) \geqslant 3$ unless stated otherwise explicitly.

Example 2.2. The tetrahedron (or 3-wheel) is the full graph on four vertices and six edges (enumerated in the ascending order: $12=I, \ldots, 34=V I)$,

$$
\boldsymbol{\gamma}_{3}=12 \wedge 13 \wedge 14 \wedge 23 \wedge 24 \wedge 34=I \wedge \cdots \wedge V I=\bigwedge_{3}^{2}
$$

This graph is nonzero. (The axis vertex is labelled 4 in this figure.)

\footnotetext{
${ }^{\mathrm{c}}$ The first example of practical calculations of the graph cohomology - with respect to the edge contracting differentialis found in [1]; a wide range of vertex-edge bi-degrees is considered there.

${ }^{\mathrm{d}}$ The vector space of graphs under study is infinite dimensional; however, it is endowed with the bi-grading (\#vertices, \#edges) so that all the homogeneous components are finite dimensional.
} 
Example 2.3. The linear combination $\boldsymbol{\gamma}_{5}$ of two 6-vertex 10-edge graphs, namely, of the pentagon wheel and triangular prism with one extra diagonal (here, $12=I$ and so on),

$$
\begin{aligned}
\gamma_{5}=12 \wedge 23 \wedge 34 \wedge 45 \wedge 51 \wedge 16 \wedge 26 \wedge 36 \wedge & 46 \wedge 56 \\
& +\frac{5}{2} \cdot 12 \wedge 23 \wedge 34 \wedge 41 \wedge 45 \wedge 15 \wedge 56 \wedge 36 \wedge 26 \wedge 13
\end{aligned}
$$

is drawn in Fig. 1 on p. 162 below (cf. [1]).

Let $\gamma_{1}$ and $\gamma_{2}$ be connected non-oriented graphs. The definition of insertion $\gamma_{1} \circ_{i} \gamma_{2}$ of the entire graph $\gamma_{1}$ into vertices of $\gamma_{2}$ and the construction of Lie bracket $[\cdot, \cdot]$ of graphs and differential $\mathrm{d}$ in the non-oriented graph complex, referring to a sign convention, are as follows (cf. [12] and $[8,14,16]$ ); these definitions apply to sums of graphs by linearity.

Definition 2. The insertion $\gamma_{1} \circ_{i} \gamma_{2}$ of an $n_{1}$-vertex graph $\gamma_{1}$ with ordered set of edges $\mathrm{E}\left(\gamma_{1}\right)$ into a graph $\gamma_{2}$ with $\# \mathrm{E}\left(\gamma_{2}\right)$ edges on $n_{2}$ vertices is a sum of graphs on $n_{1}+n_{2}-1$ vertices and $\# \mathrm{E}\left(\gamma_{1}\right)+$ $\# \mathrm{E}\left(\gamma_{2}\right)$ edges. Topologically, the sum $\gamma_{1} \circ_{i} \gamma_{2}=\sum\left(\gamma_{1} \rightarrow v\right.$ in $\left.\gamma_{2}\right)$ consists of all the graphs in which a vertex $v$ from $\gamma_{2}$ is replaced by the entire graph $\gamma_{1}$ and the edges touching $v$ in $\gamma_{2}$ are re-attached to the vertices of $\gamma_{1}$ in all possible ways. ${ }^{\mathrm{e}}$ By convention, in every new term the edge ordering is $\mathrm{E}\left(\gamma_{1}\right) \wedge \mathrm{E}\left(\gamma_{2}\right)$.

To simplify sums of graphs, first eliminate the zero graphs. Now suppose that in a sum, two nonoriented graphs, say $\alpha$ and $\beta$, are isomorphic (topologically, i.e. regardless of the respective vertex labellings and edge orderings $\mathrm{E}(\alpha)$ and $\mathrm{E}(\beta))$. By using that isomorphism, which establishes a 11 correspondence between the edges, extract the sign from the equation $\mathrm{E}(\alpha)= \pm \mathrm{E}(\beta)$. If " + ", then $\alpha=\beta$; else $\alpha=-\beta$. Collecting similar terms is now elementary.

Lemma 2.1. The bi-linear graded skew-symmetric operation,

$$
\left[\gamma_{1}, \gamma_{2}\right]=\gamma_{1} \circ_{i} \gamma_{2}-(-)^{\# E\left(\gamma_{1}\right) \cdot \# E\left(\gamma_{2}\right)} \gamma_{2} \circ_{i} \gamma_{1}
$$

is a Lie bracket on the vector space $\mathfrak{G}$ of non-oriented graphs. ${ }^{\mathrm{f}}$

Lemma 2.2. The operator $\mathrm{d}($ graph $)=[\bullet \bullet$, graph $]$ is a differential: $\mathrm{d}^{2}=0$.

In effect, the mapping $\mathrm{d}$ blows up every vertex $v$ in its argument in such a way that whenever the number of adjacent vertices $N(v) \geqslant 2$ is sufficient, each end of the inserted edge $\bullet \bullet$ is connected with the rest of the graph by at least one edge.

Theorem 1 ( [12]). The real vector space $\mathfrak{G}$ of non-oriented graphs is a differential graded Lie algebra $($ dgLa) with Lie bracket $[\cdot, \cdot]$ and differential $\mathrm{d}=[\bullet \bullet, \cdot]$. The differential $\mathrm{d}$ is a graded derivation of the bracket $[\cdot, \cdot]$ (due to the Jacobi identity for this Lie algebra structure).

${ }^{\mathrm{e}}$ Let the enumeration of vertices in every such term in the sum start running over the enumerated vertices in $\gamma_{2}$ until $v$ is reached. Now the enumeration counts the vertices in the graph $\gamma_{1}$ and then it resumes with the remaining vertices (if any) that go after $v$ in $\gamma_{2}$.

${ }^{\mathrm{f}}$ The postulated precedence or antecedence of the wedge product of edges from $\gamma_{1}$ with respect to the edges from $\gamma_{2}$ in every graph within $\gamma_{1} \circ_{i} \gamma_{2}$ produce the operations $\circ_{i}$ which coincide with or, respectively, differ from Definition 2 by the sign factor $(-)^{\# E\left(\gamma_{1}\right) \cdot \# E\left(\gamma_{2}\right)}$. The same applies to the Lie bracket of graphs $\left[\gamma_{1}, \gamma_{2}\right]$ if the operation $\gamma_{1} \circ_{i} \gamma_{2}$ is the insertion of $\gamma_{2}$ into $\gamma_{1}$ (as in [14]). Anyway, the notion of d-cocycles which we presently recall is well defined and insensitive to such sign ambiguity. 
The graphs $\boldsymbol{\gamma}_{3}$ and $\boldsymbol{\gamma}_{5}$ from Examples 2.2 and 2.3 are d-cocycles (this will be shown in $\S 3$ ). Therefore, their commutator $\left[\boldsymbol{\gamma}_{3}, \boldsymbol{\gamma}_{5}\right]$ is also in kerd. Neither $\boldsymbol{\gamma}_{3}$ nor $\boldsymbol{\gamma}_{5}$ is exact, hence marking a nontrivial cohomology class in the non-oriented graph complex.

Theorem 2 ( [8, Th. 5.5]). At every $\ell \in \mathbb{N}$ in the connected graph complex there is a nontrivial $\mathrm{d}$-cocycle on $2 \ell+1$ vertices and $4 \ell+2$ edges. Such cocycle contains the $(2 \ell+1)$-wheel in which, by definition, the axis vertex is connected with every other vertex by a spoke so that each of those $2 \ell$ vertices is adjacent to the axis and two neighbours; the cocycle marked by the $(2 \ell+1)$-wheel graph can contain other $(2 \ell+1,4 \ell+2)$-graphs.

Example 2.4. For $\ell=3$ the heptagon wheel cocycle $\gamma_{7}$, which we present in this paper, consists of the heptagon-wheel graph on $(2 \cdot 3+1)+1=8$ vertices and $2(2 \cdot 3+1)=14$ edges and forty-five other graphs with equally many vertices and edges (hence of the same number of generators of their homotopy groups, or basic loops: $7=14-(8-1)$ ), and with real coefficients. All these weighted graphs are drawn in Appendix A (see pp. 169-172). The chosen - lexicographic - ordering of edges in each term is read from the encoding of every such graph (see also Table 1 on p. 166; each entry of that table is a listing $I \prec \cdots \prec X I V$ of the ordered edge set, followed by the coefficient of that graph). A verification of the cocycle condition $\mathrm{d}\left(\boldsymbol{\gamma}_{7}\right)=0$ for this solution is computer-assisted; it has been performed by using the code (in SAGE programming language) which is contained in Appendix B.

\section{Calculating the differential of graphs}

Example 3.1 $\left(\mathrm{d} \boldsymbol{\gamma}_{3}=0\right)$. The tetrahedron $\boldsymbol{\gamma}_{3}$ is the full graph on $n=4$ vertices; we are free to choose any ordering of the six edges in it, so let it be lexicographic:

$$
\mathrm{E}\left(\boldsymbol{\gamma}_{3}\right)=12 \wedge 13 \wedge 14 \wedge 23 \wedge 24 \wedge 34=I \wedge I I \wedge I I I \wedge I V \wedge V \wedge V I
$$

The differential of this graph is equal to

$$
\mathrm{d}\left(\boldsymbol{\gamma}_{3}\right)=\left[\bullet \bullet, \boldsymbol{\gamma}_{3}\right]=\bullet \bullet \circ_{i} \boldsymbol{\gamma}_{3}-(-)^{\# E(\bullet \bullet) . \# E\left(\boldsymbol{\gamma}_{3}\right)} \boldsymbol{\gamma}_{3} \circ_{i} \bullet \bullet=\bullet \bullet \circ_{i} \boldsymbol{\gamma}_{3}-\boldsymbol{\gamma}_{3} \circ_{i} \bullet \bullet,
$$

since $\# E\left(\boldsymbol{\gamma}_{3}\right)=6$. Note that every vertex of valency one appears twice in $\mathrm{d}\left(\boldsymbol{\gamma}_{3}\right)$ : namely in the minuend (where the edge ordering is $E \wedge I \wedge \cdots \wedge V I$ by definition of $\circ_{i}$ ) and subtrahend (where the edge ordering is $I \wedge \cdots \wedge V I \wedge E$ ). Because these edge orderings differ by a parity-even permutation, such graphs in $\bullet \circ_{i} \gamma_{3}$ and $\gamma_{3} \circ_{i} \bullet$ carry the same sign. Hence they cancel in the difference $\bullet \bullet \circ_{i} \gamma_{3}-\boldsymbol{\gamma}_{3} \circ_{i} \bullet \bullet$, and no longer shall we pay any attention to the leaves, absent in the differential of any graph. It is readily seen that the twenty-four graphs $\left(24=4\right.$ vertices $\cdot\left(\begin{array}{l}3 \\ 1\end{array}\right) \cdot 2$ ends of $\left.\bullet \bullet\right)$ we are left with in $\mathrm{d}\left(\boldsymbol{\gamma}_{3}\right)$ are of the shape drawn here. A vertex is blown up to the new edge $E=\bullet \bullet$

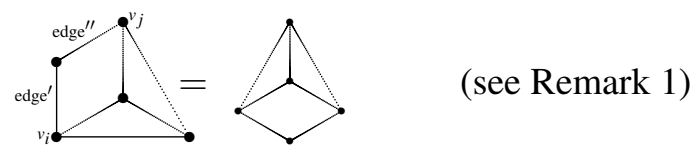

whose ends are both attached to the rest of the graph along the old edges. This shape can be obtained in two ways: by blowing up $v_{i}$, so that edge' is the newly inserted edge, or by blowing up $v_{j}$, so that edge ${ }^{\prime \prime}$ is the newly inserted edge. By Lemma 3.1 below we conclude that $\mathrm{d}\left(\boldsymbol{\gamma}_{3}\right)=0$. 
Remark 1. Incidentally, every graph which was obtained in $\mathrm{d}\left(\boldsymbol{\gamma}_{3}\right)$ itself is a zero graph. Indeed, it is symmetric with respect to a flip over the vertical line and this symmetry swaps three edge pairs (see Definition 1).

Lemma 3.1 (handshake). In the differential of any graph $\gamma$ such that the valency of all vertices in $\gamma$ is strictly greater than two, the graphs in which one end of the newly inserted edge $\bullet \bullet$ has valency two, all cancel.

Proof. Let $v$ be such a vertex in $\mathrm{d}(\gamma)$, i.e. the vertex $v$ is an end of the inserted edge $\bullet \bullet$ and it has

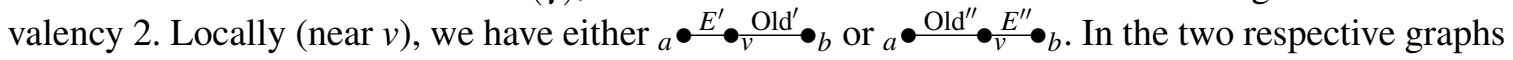
in $\mathrm{d}(\gamma)$ the rest, consisting only of old edges and vertices of valency $\geqslant 3$ from $\gamma$, is the same. Yet the two graphs are topologically equal; furthermore, they have the same ordering of edges except for $E^{\prime}=\mathrm{Old}^{\prime \prime}$ and $\mathrm{Old}^{\prime}=E^{\prime \prime}$. Recall that by construction, the edge ordering of the first graph is $E^{\prime} \wedge \cdots \wedge \operatorname{Old}^{\prime} \wedge \cdots$, whereas for the second graph it is $E^{\prime \prime} \wedge \cdots \wedge \operatorname{Old}^{\prime \prime} \wedge \cdots$; the new edge always goes first. So effectively, two edges are swapped. Therefore,

$$
E^{\prime \prime} \wedge \cdots \wedge \operatorname{Old}^{\prime \prime} \wedge \cdots=\operatorname{Old}^{\prime} \wedge \cdots \wedge E^{\prime} \wedge \cdots=-E^{\prime} \wedge \cdots \wedge \operatorname{Old}^{\prime} \wedge \cdots .
$$

Hence in every such pair in $\mathrm{d}(\gamma)$, the graphs occur with opposite signs. Moreover, the initial hypothesis $N(a) \geqslant 3$ about the valency of all vertices $a$ in the graph $\gamma$ guarantees that the cancelling pairs of graphs in $\mathrm{d}(\gamma)$ do not intersect, $\stackrel{\mathrm{g}}{\text { and thus all cancel. }}$

Corollary 3 (to Lemma 3.1). In the differential of any graph with vertices of valency $>2$, the blow up of a vertex of valency 3 produces only the handshakes, that is the graphs which cancel out by Lemma 3.1 ( cf. footnote i on p. 167 below).

Example 3.2 $\left(\mathrm{d} \boldsymbol{\gamma}_{5}=0\right)$. The pentagon-wheel cocycle is the sum of two graphs with real coefficients which is drawn in Fig. 1. The edges in every term are ordered by $I \wedge \cdots \wedge X$. The differential of a sum

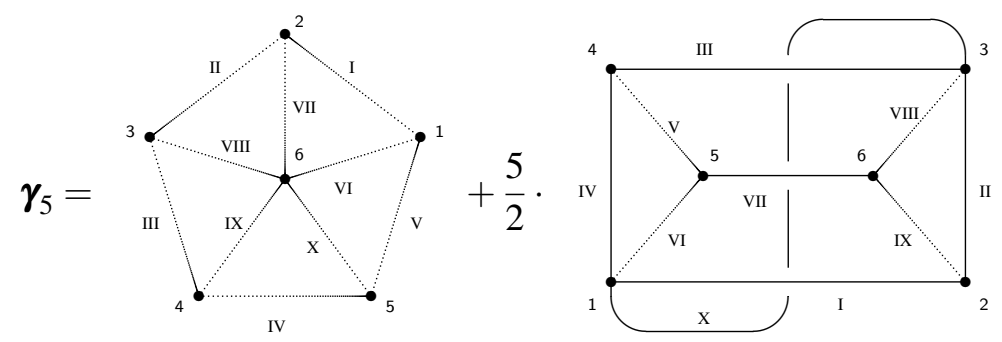

Fig. 1. The Kontsevich-Willwacher pentagon-wheel cocycle $\boldsymbol{\gamma}_{5}$.

of graphs is the sum of their differentials; this is why we calculate them separately and then collect similar terms. By the above, neither contains any leaves; likewise by the handshake Lemma 3.1, all the graphs - in which a new vertex (of valency 2) appears as midpoint of the already existing edge cancel. By Corollary 3 it remains for us to consider the blow-ups of only the vertices of valency $\geqslant 4$ (cf. [12]). Such are the axis vertex of the pentagon wheel and vertices labelled 1 and 3 in the other

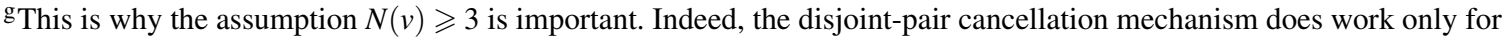
chains with even numbers of valency-two vertices $v$ in $\gamma$. Here is an example (of one such vertex $v$ between $a$ and $b$ ) when it actually does not: in the differential of a graph that contains $a_{a} I^{I} \bullet_{v}^{I I} \bullet b$, we locally obtain $a \bullet \bullet_{a}{ }^{\prime} \bullet_{v}^{I I} \bullet_{b}+{ }_{a} \bullet \bullet_{v}{ }_{v}^{E} \bullet \bullet_{v}^{I I} \bullet_{b}$ $+{ }_{a} \cdot{ }^{I} \cdot{ }_{v}^{I I} \bullet b_{b}^{\prime} \bullet$, so that the middle term can be cancelled against either the first or the last one but not with both of them simultaneously.
} 
graph (the prism). By blowing up the pentagon wheel axis we shall obtain the (nonzero) 'human' and the (zero) 'monkey' graphs, presented in what follows. Likewise from the prism graph in $\boldsymbol{\gamma}_{5}$ one obtains the 'human', the 'monkey', and the (zero) 'stone'. Let us now discuss this in full detail.

From the pentagon wheel we obtain 2.5 Da Vinci's 'human' graphs, two of which are portrayed in Fig. 2. (The factor 2 occurs from the two distinct ways to attach three versus two old edges in the wheel to the loose ends of the inserted edge $\bullet-\bullet$.) We claim that all the five 'human' graphs

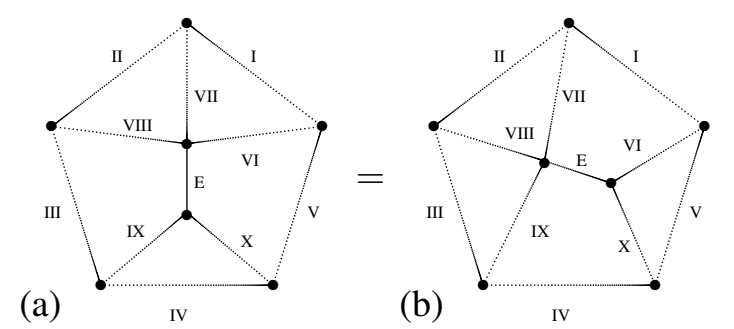

Fig. 2. Two of the fourteen Da Vinci's 'human' graphs occurring with weights in $\mathrm{d} \boldsymbol{\gamma}_{5}$.

(i.e. standing with their feet on the edges $I, \ldots, V$ in the pentagon wheel) carry the same sign, providing the overall coefficient $+10=2 \cdot(+5)$ of such graph in the differential of the wheel. The graph (b) is topologically equal to the graph (a); indeed, the matching of their edges is $I^{(b)}=V^{(a)}$, $I I^{(b)}=I^{(a)}, I I I^{(b)}=I I^{(a)}, I V^{(b)}=I I I^{(a)}, V^{(b)}=I V^{(a)}, V I^{(b)}=X^{(a)}, V I I^{(b)}=V I^{(a)}, V I I I^{(b)}=V I I^{(a)}$, $I X^{(b)}=V I I I^{(a)}$, and $X^{(b)}=I X^{(a)}$; also $E^{(b)}=E^{(a)}$. Hence the postulated ordering of edges in (b) is

$$
\begin{aligned}
E^{(b)} \wedge I^{(b)} \wedge \cdots \wedge X^{(b)}= & E^{(a)} \wedge V^{(a)} \wedge I^{(a)} \wedge I I^{(a)} \wedge I I I^{(a)} \wedge I V^{(a)} \wedge \\
& \wedge X^{(a)} \wedge V I^{(a)} \wedge V I I^{(a)} \wedge V I I I^{(a)} \wedge I X^{(a)}=+E^{(a)} \wedge I^{(a)} \wedge \cdots \wedge X^{(a)},
\end{aligned}
$$

which equals the edge ordering of the graph (a). For the other three graphs of this shape the equalities of wedge products are similar: a parity-even permutation of edges works out the mapping of graphs, e.g., to the graph (a) which we take as the reference.

From the pentagon wheel we also obtain 2.5 'monkey' graphs, a specimen of which is shown in Fig. 3 below. Note that the 'monkey' graph is mirror-symmetric, see the redrawing. This symmetry

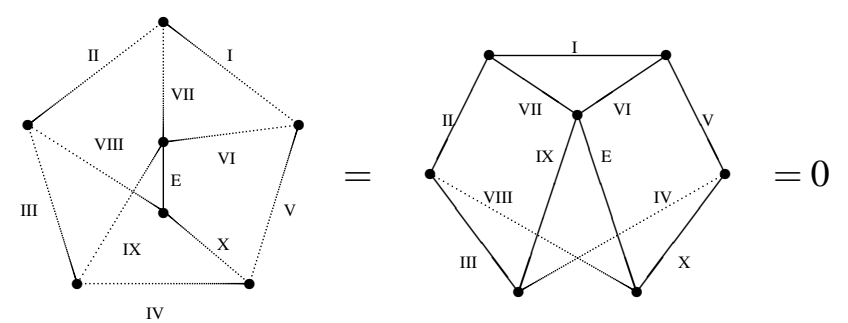

Fig. 3. The 'monkey' graph: animal touches earth with its palm; this is an example of zero graph.

induces a permutation of edges which swaps 5 pairs, so (since 5 is odd) the 'monkey' graph is equal to zero.

Now consider the graphs obtained by blowing up vertices 1 and 3 in the prism graph. How are the four old neighbors distributed over the ends of the inserted edge? Whenever those four old neighbours are distributed in proportion $4=3+1$ (i.e. with valencies 4 and 2 for the two ends of 
the inserted edge), there is no contribution from the resulting graphs to $\mathrm{d}$ (prism) by the handshake Lemma 3.1. So the graphs which could contibute are only those with the $4=2+2$ distribution (i.e. with valency 3 for either of the ends of the inserted edge). For one fixed neighbour of one of the new edge's ends there are three ways to choose the second neighbour of that vertex. This is how the 'human', 'monkey', and 'stone' graphs are presently obtained.

Let us blow up vertex 1 in the prism in these three different ways. First we make the end (now marked 1) of the inserted edge adjacent to 2 and 3, and the other end (marked $1^{\prime}$ ) to vertices 4 and 5; the resulting graph is the 'human' graph shown in Fig. 4. From the prism graph we obtain $2 \cdot 2=4$

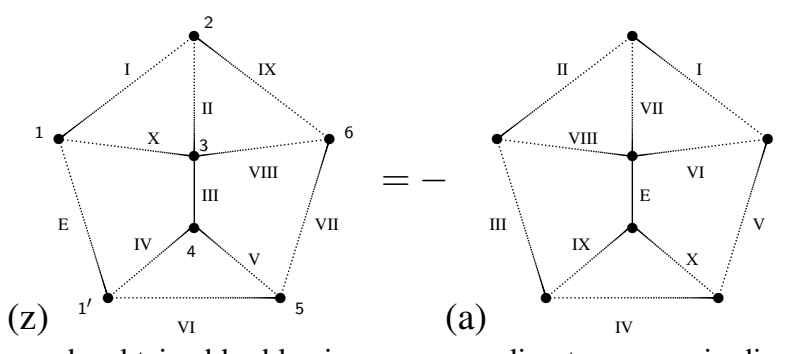

Fig. 4. One of the 'human' graphs obtained by blowing up - according to a scenario discussed in the text - a vertex of valency four in the prism graph from $\boldsymbol{\gamma}_{5}$.

such 'human' graphs. One of the factors 2 is obtained like before, namely by attaching a given set of old edges to one or the other end of the inserted edge $\bullet \bullet$, see p. 163; the other factor 2 comes by the rotational symmetry of the prism graph. Indeed, the prism with one diagonal is symmetric under the rotation by angle $\pi$ that transposes the vertices $1 \rightleftarrows 3,2 \rightleftarrows 4$, and $5 \rightleftarrows 6$. This is why the same 'human' graph is obtained when the vertex 3 is blown up according to a similar scenario. We claim that the permutation of edges that relates the two graphs is parity-even (similar to (3.1)), so they do not cancel but add up. Summarizing, the overal coefficient of the 'human' graph - produced in d(prism) for the edge ordering $E \wedge I \wedge \cdots \wedge X$ shown in Fig. 4 - equals $2 \cdot 2=+4$.

The count of an overall contribution $10+\frac{5}{2} \cdot(+4) \cdot(-1$ from edge ordering $)=0$ to the differential $\mathrm{d}\left(\boldsymbol{\gamma}_{5}\right)$ of the cocycle $\boldsymbol{\gamma}_{5}$ will be performed using Eq. (3.2); right now let us inspect the vanishing of contributions from the other two types of graphs wich are obtained by the two possible edge distribution scenarios (with respect to the ends of the new edge $\bullet \bullet$ that replaces the blown-up vertex 1 or 3 in the prism).

The 'monkey' graph is obtained by blowing up the vertex 1 (or 3) in the prism and then attaching the new edge's end, still marked 1 , to the vertices 2 and 4 . The other end, now marked $1^{\prime}$, of the new edge becomes adjacent to the vertices 3 and 5. We keep in mind that every 'monkey' graph itself is equal to zero, hence no contribution to $\mathrm{d}$ (prism) occurs.

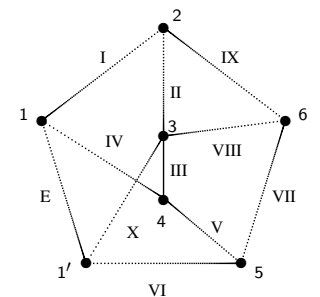

So far, the new vertex 1 has always been a fixed neighbour of vertex 2 , and it was made adjacent to 3 in the 'human' and to 4 in the 'monkey' graphs, respectively. The overall set of neigbours of the new edge $1-1^{\prime}$, apart from the fixed vertex 2 , consists of vertices 3,4 and 5 . So the third scenario to consider is the 'stone' graph (see Fig. 5) in which the new vertex 1 is adjacent to $1^{\prime}$, 2 , and 5 , whereas the new vertex $1^{\prime}$ neighbours 1,3 , and 4 . This graph is mirror-symmetric under the transposition of vertices $1^{\prime} \rightleftarrows 2$ and $4 \rightleftarrows 6$, which induces the swaps in five edge pairs, namely, $I I \rightleftarrows I I I, E \rightleftarrows X, V I \rightleftarrows V I I I, V \rightleftarrows I X$, and $I \rightleftarrows I V$. Arguing as before, we deduce that every such 'stone' graph (obtained by a blow up of either 1 or 3 in the prism) is zero. 
R. Buring et al. / The heptagon-wheel cocycle in the graph complex

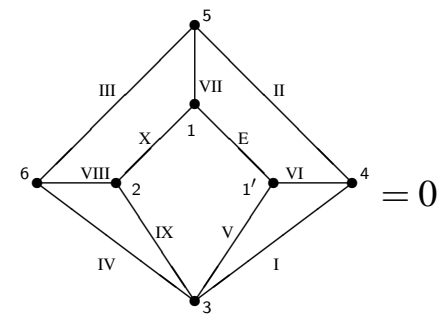

Fig. 5. The 'stone' graph: the new vertex 1 is adjacent to $1^{\prime}, 2$, and 5 .

Our final task in the calculation of $\mathrm{d}\left(\boldsymbol{\gamma}_{5}\right)$ is collecting the coefficients of the 'human' graphs from d(5-wheel) and d(prism), coming not only with coefficients 10 and 4 respectively, but also with the respective edge orderings. To discriminate edges between the two pictures, that is originating from the pentagon wheel and the prism, let us use the superscripts $(a)$ and $(z)$, see Fig. 4. The edge matching is $E^{(z)}=I I I^{(a)}, I^{(z)}=I I^{(a)}, I I^{(z)}=V I I^{(a)}, I I I^{(z)}=E^{(a)}, I V^{(z)}=I X^{(a)}, V^{(z)}=X^{(a)}$, $V I^{(z)}=I V^{(a)}, V I I^{(z)}=V^{(a)}, V I I I^{(z)}=V I^{(a)}, I X^{(z)}=I^{(a)}$, and $X^{(z)}=V I I I^{(a)}$. Consequently, for the edge orderings we have

$$
\begin{aligned}
& E^{(z)} \wedge I^{(z)} \wedge \cdots \wedge X^{(z)}= \\
& \quad I I I^{(a)} \wedge I I^{(a)} \wedge V I I^{(a)} \wedge E^{(a)} \wedge I X^{(a)} \wedge X^{(a)} \wedge I V^{(a)} \wedge V^{(a)} \wedge V I^{(a)} \wedge I^{(a)} \wedge V I I I^{(a)} \\
& =(-)^{23} E^{(a)} \wedge I^{(a)} \wedge \cdots \wedge X^{(a)} .
\end{aligned}
$$

This argument shows that the graph differential of the linear combination $(+1) \cdot$ pentagon-wheel + $\frac{5}{2}$. prism, with either graph's edge ordering specified as in Example 2.3, vanishes. In other words, $\boldsymbol{\gamma}_{5}$ is a d-cocycle.

\section{A representative of the heptagon-wheel cocycle $\boldsymbol{\gamma}_{7}$}

It is already known that the heptagon-wheel cocycle $\boldsymbol{\gamma}_{7}$, the existence of which was stated in Theorem 2, is unique modulo d-trivial terms in the respective cohomology group of connected graphs on 8 vertices and 14 edges (hence with 7 basic loops), cf. [14].

Theorem 4. The encoding of every term in a representative of the cocycle $\boldsymbol{\gamma}_{7}$ is given in Table 1 , the format of lines in which is the lexicographic-ordered list of fourteen edges I $\wedge \cdots \wedge X I V$ followed by the nonzero real coefficient. The forty-six graphs that form this representative of the $\mathrm{d}$-cohomology class $\boldsymbol{\gamma}_{7}$ are shown on pages 169-172.

Proof scheme. This reasoning is computer-assisted. First, all connected graphs on 8 vertices and 14 edges, and without multiple edges were generated. (There are 1579 such graphs; note that arbitrary valency $N(v) \geqslant 1$ of vertices was allowed.) The coefficient of the heptagon wheel was set equal to +1 , all other coefficients still to be determined. After calculating the differential of the sum of all these weighted graphs (we used a program in S AGE, see Appendix B), zero graphs were eliminated and the remaining terms were collected (in the same way as is explained in $\S 3$ ). In the resulting sum of weighted graphs on 9 vertices and 15 edges, we equated each coefficient to zero. We solved this linear algebraic system w.r.t. the coefficients of graphs in $\boldsymbol{\gamma}_{7}$. There are $N_{\mathrm{im}}(7)=35$ free parameters in the general solution; such parameters count the coboundaries which cannot modify the cohomology class marked by any particular representative (see Table 2 on p. 167 below). Therefore the solution $\boldsymbol{\gamma}_{7}$ is unique modulo d-exact terms. All those free parameters are now set to zero and the resulting nonzero values of the graph coefficients are listed in Table 1. 
R. Buring et al. / The heptagon-wheel cocycle in the graph complex

Table 1. The heptagon-wheel graph cocycle $\boldsymbol{\gamma}_{7}$.

\begin{tabular}{lcccc}
\hline Graph encoding & Coeff. & Graph encoding & Coeff. \\
\hline 1617182325283438464857586878 & 1 & 1213182526373845464756576878 & -7 \\
1214182327353746485758676878 & $-21 / 8$ & 1214162325363745485758676878 & $77 / 8$ \\
1314182325283746485657676878 & $-77 / 4$ & 1316172425263537454858676878 & -7 \\
1213152427353646485758676878 & $-35 / 8$ & 1415172326283738464856576878 & $49 / 4$ \\
1213182426373846475657586878 & $49 / 8$ & 1216182728343638464756575878 & $-147 / 8$ \\
1417182325263537464856586778 & $77 / 8$ & 1215162728353638454647576878 & $-21 / 8$ \\
1213182627353845464756576878 & $-105 / 8$ & 1214182327353645465758676878 & $-35 / 8$ \\
1214182327363846485657586778 & $7 / 8$ & 1415162326283738464857586778 & $-49 / 4$ \\
1214152327353646485758676878 & $35 / 8$ & 1215182328343746485657676878 & $105 / 8$ \\
1213142728363846475657586878 & $-49 / 8$ & 1214172326373846485657586878 & $-49 / 8$ \\
1213182527343647485658676878 & $35 / 4$ & 1216182527353637454648576878 & $49 / 16$ \\
1213142526363845475758676878 & $-119 / 16$ & 1213182527353646474856576878 & 7 \\
1213152428363847485657676878 & $49 / 8$ & 1214182528343638475758676878 & -7 \\
1213142328374648565758676878 & $77 / 4$ & 1216182527353637454648586778 & $-77 / 16$ \\
1215172526353638454748676878 & $-49 / 8$ & 1214182327353846475758676878 & $77 / 4$ \\
1315182426283738464756576878 & $-49 / 4$ & 1214152327363846485758676878 & $35 / 2$ \\
1314182526283638474856576778 & $-49 / 4$ & 1213182527343646485758676878 & $-105 / 8$ \\
1214182328353746485657676878 & -7 & 1215162527353638464748576878 & -7 \\
1214182328363846475657586778 & -7 & 1213162528343747485758676878 & $-147 / 16$ \\
1215162527353638464748586778 & $49 / 8$ & 1213172526353745464858676878 & $-77 / 4$ \\
1214182328363746475657586878 & $49 / 8$ & 1214172327353846485758676878 & $-49 / 8$ \\
1213152627353645474858676878 & -7 & 1213152628353745464758676878 & $-7 / 4$ \\
1213182428353846475758676878 & 7 & 1214182326363847485657586778 & -7 \\
\hline
\end{tabular}

Proposition 5 (see [14, Table 1]). The space of nontrivial d-cocycles which are built of connected graphs on $n$ vertices and $2 n-2$ edges at $1 \leqslant n \leqslant 9$ is spanned by the terahedron $\boldsymbol{\gamma}_{3}$, pentagonwheel cocycle $\boldsymbol{\gamma}_{5}$ that consists of two graphs (see Example 2.3), heptagon-wheel cocycle $\boldsymbol{\gamma}_{7}$ from Theorem 4 , and the Lie bracket $\left[\boldsymbol{\gamma}_{3}, \boldsymbol{\gamma}_{5}\right]$. At the same time, for either $n=5$ or $n=7$, the respective graph cohomology groups are trivial. ${ }^{\mathrm{h}}$

Verification. The dimension $N_{\mathrm{ker}}$ of the space of cocycles built of connected graphs $\boldsymbol{\gamma}$ on $n$ vertices and $2 n-2$ edges is equal to the number of free parameters in the general solution to the linear system $\mathrm{d}$ (sum of such graphs $\gamma$ with undetermined coefficients $)=0$. At the same time, to determine the dimension $N_{\text {im }}$ of the subspace of coboundaries $\gamma=\mathrm{d}(\delta)$, i.e. of those cocycles which are the differentials of connected graphs on $n-1$ vertices and $2 n-3$ edges, we first count the number of $N_{\delta}$ of nonzero connected graphs $\delta$ in that vertex-edge bi-grading. Then we subtract from $N_{\delta}$ the number $N_{0}$ of free parameters in the general solution to the linear algebraic system $\mathrm{d}$ (sums of such graphs $\delta$ with undetermined coefficients) $=0$. This subtrahend counts the number of relations between exact terms $\gamma=\mathrm{d}(\boldsymbol{\delta})$; for $n<9$ it is zero. The dimension of cohomology group $H^{*}(n)$ in bi-grading $(n, 2 n-2)$ is then $N_{\mathrm{ker}}-N_{\mathrm{im}}=N_{\mathrm{ker}}-\left(N_{\delta}-N_{0}\right)$.

Our present count of the overall number of connected graphs (and of the zero graphs among them) and the dimensions $N_{\mathrm{ker}}, N_{\delta}, N_{0}$ and $N_{\mathrm{im}}$ of the respective vector spaces are summarized in Tables 2 and 3.

\footnotetext{
${ }^{\mathrm{h}}$ None of the results in Theorem 4 and Proposition 5 involves floating point operations in the way how it is obtained; hence even if computer-assisted, both the claims are exact.
} 
Table 2. Dimensions of connected graph spaces and cohomology groups.

\begin{tabular}{|c|c|c|c|c|c|c|c|c|c|c|}
\hline \multicolumn{2}{|c|}{$n$} & $\# E$ & $\#($ graphs $)$ & $\#(=0)$ & \multicolumn{2}{c|}{$\#(\neq 0), N_{\delta}$} & \multicolumn{2}{|c|}{$N_{\mathrm{ker}}, N_{0}$} & $N_{\mathrm{im}}$ & $\operatorname{dim} H^{*}(n)$ \\
\hline 4 & & 6 & 1 & 0 & 1 & & 1 & & & 1 \\
& 3 & 5 & 0 & - & - & - & & - & - & \\
\hline 5 & & 8 & 2 & 2 & 0 & & - & & & 0 \\
& 4 & 7 & 0 & - & - & - & & - & - & \\
\hline 6 & & 10 & 14 & 8 & 6 & & 1 & & & 1 \\
& 5 & 9 & 1 & 1 & - & 0 & & - & - & \\
\hline 7 & & 12 & 126 & 78 & 48 & & 1 & & & 0 \\
& 6 & 11 & 9 & 8 & - & 1 & & 0 & 1 & \\
\hline 8 & & 14 & 1579 & 605 & 974 & & 36 & & & 1 \\
& 7 & 13 & 95 & 60 & - & 35 & & 0 & 35 & \\
\hline 9 & & 16 & 26631 & 7557 & 19074 & & 883 & & & 1 \\
& 8 & 15 & 1515 & 602 & - & 913 & & 31 & 882 & \\
\hline
\end{tabular}

Remark 2. This reasoning covers all the connected graphs with specified number of vertices and edges, meaning that the valency $N(v)$ of every graph vertex $v$ can be any positive number (if $n>1$ ). By Lemma 3.1 on p. 162 it is seen that for the subspaces $V_{>2}$ of connected graphs restricted by $N(v)>2$ for all $v$, the inclusion $\mathrm{d}\left(V_{>2}\right) \subseteq V_{>2}$ holds. Therefore, the dimensions of cohomology groups for graphs with such restriction on valency cannot exceed the dimension of respective cohomology groups for all the graphs under study (i.e. $N(v)>0$ ). ${ }^{i}$ This means that trivial cohomology groups remain trivial under the extra assumption $N(v)>2$ on valency; yet we already know the generators $\boldsymbol{\gamma}_{3}, \boldsymbol{\gamma}_{5}, \boldsymbol{\gamma}_{7}$, and $\left[\boldsymbol{\gamma}_{3}, \boldsymbol{\gamma}_{5}\right]$ of all the nontrivial cohomology groups at $n \leqslant 9$. This is confirmed in Table 3 .

Table 3. Dimensions of connected graph spaces with $N(v)>2$ and dimensions of cohomology groups in bi-degree $(n, 2 n-$ 2).

\begin{tabular}{|c|c|c|c|c|c|c|c|c|c|c|}
\hline \multicolumn{2}{|c|}{$n$} & $\# E$ & $\#($ graphs $)$ & $\#(=0)$ & $\#(\neq 0), N_{\delta}$ & \multicolumn{2}{|c|}{$N_{\text {ker }}, N_{0}$} & $N_{\text {im }}$ & $\operatorname{dim} H^{*}(n)$ \\
\hline 4 & & 6 & 1 & 0 & 1 & & 1 & & & 1 \\
& 3 & 5 & 0 & - & - & - & & - & - & \\
\hline 5 & & 8 & 1 & 1 & 0 & & - & & & 0 \\
& 4 & 7 & 0 & - & - & - & & - & - & \\
\hline 6 & & 10 & 4 & 2 & 2 & & 1 & & & 1 \\
& 5 & 9 & 1 & 1 & - & 0 & & - & - & \\
\hline 7 & & 12 & 18 & 12 & 6 & & 1 & & & 0 \\
& 6 & 11 & 5 & 4 & - & 1 & & 0 & 1 & \\
\hline 8 & & 14 & 136 & 61 & 75 & & 11 & & & 1 \\
& 7 & 13 & 30 & 20 & - & 10 & & 0 & 10 & \\
\hline 9 & & 16 & 1377 & 498 & 879 & & 164 & & & 1 \\
& 8 & 15 & 309 & 130 & - & 179 & & 16 & 163 & \\
\hline
\end{tabular}

${ }^{\mathrm{i}}$ Indeed, we recall that these cohomology dimensions - in the count with versus without restriction $N(v)>2$ of the valency - are the same (e.g., see [16, Proposition 3.4] with a sketch of the proof). 
We finally note that the numbers of nonzero graphs with a specified number of vertices and edges (and $N(v)>2$ ), which we list in Table 3, all coincide with the respective entries in Table II in the paper [17].

Remark 3. We expect that there are many d-cocycles on $n$ vertices and $2 n-2$ edges other than the ones containing the $(2 \ell+1)$-wheel graphs (which Theorem 2 provides) or their iterated commutators. Namely, some terms in a weighted sum $\gamma \in$ kerd can be disjoint graphs; moreover, the vertex-edge bi-grading of a connected component of a given term can be other than $(m, 2 m-2)$ for $m \in \mathbb{N}$. Indeed, for any tuple of d-cocycles $\gamma_{i}$ on $n_{i}$ vertices and $E_{i}$ edges satisfying $\sum_{i} n_{i}=n$ and $\sum_{i} E_{i}=2 n-2$, one has that $\gamma:=\bigsqcup_{i} \gamma_{i} \in$ kerd. The graphs $\gamma_{i}$ can be restricted by a requirement that each of them belongs to the domain of the orientation mapping $\mathrm{O} \vec{r}$, so that $\mathrm{O} \vec{r}(\gamma)$ is a Kontsevich bivector graph (see [12] and [2,7]). In this way new classes of generators of infinitesimal symmetries $\dot{\mathscr{P}}=\mathrm{O} \overrightarrow{\mathrm{r}}(\gamma)(\mathscr{P})$ are obtained for Poisson structures $\mathscr{P}$.

\section{Acknowledgements}

The authors are grateful to M. Kontsevich and T. Willwacher for helpful discussion; the authors thank the referees for criticism and advice. This research was supported in part by JBI RUG project 106552 (Groningen, The Netherlands). A part of this research was done while R. Buring and A. Kiselev were visiting at the IHÉS (Bures-sur-Yvette, France) and A. Kiselev was visiting at the MPIM (Bonn) and Johannes Gutenberg-Universität in Mainz, Germany.

\section{References}

[1] D. Bar-Natan and B.D. McKay, Graph cohomology - An overview and some computations, 13 p. (2001, unpublished), http://www.math.toronto.edu/ drorbn/papers/GCOC/GCOC.ps

[2] A. Bouisaghouane, R. Buring and A. Kiselev, The Kontsevich tetrahedral flow revisited, J. Geom. Phys. 119 (2017) 272-285. (Preprint arXiv: 1608.01710 [q-alg])

[3] A. Bouisaghouane and A.V. Kiselev, Do the Kontsevich tetrahedral flows preserve or destroy the space of Poisson bi-vectors? J. Phys.: Conf. Ser. 804 (2017) Proc. XXIV Int. conf. 'Integrable Systems and Quantum Symmetries' (14-18 June 2016, ČVUT Prague, Czech Republic), Paper 012008, 10 p. (Preprint arXiv: 1609.06677 [q-alg])

[4] F. Brown, Mixed Tate motives over $\mathbb{Z}$, Ann. Math. (2) 175:2 (2012) 949-976.

[5] R. Buring and A.V. Kiselev, On the Kontsevich $\star$-product associativity mechanism, PEPAN Letters 14:2 (2017) 403-407. (Preprint arXiv: 1602.09036 [q-alg])

[6] R. Buring and A.V. Kiselev, The expansion $\star \bmod \bar{\sigma}\left(\hbar^{4}\right)$ and computer-assisted proof schemes in the Kontsevich deformation quantization, Preprint IHÉS/M/17/05 (2017) arXiv: 1702.00681 [math.CO], $67 \mathrm{p}$.

[7] R. Buring, A.V. Kiselev and N.J. Rutten, The Kontsevich-Willwacher pentagon-wheel symmetry of Poisson structures, SDSP IV (12-16 June 2017, ČVUT Děčín, Czech Republic).

[8] V.A. Dolgushev, C.L. Rogers and T.H. Willwacher, Kontsevich's graph complex, GRT, and the deformation complex of the sheaf of polyvector fields, Ann. Math. 182:3 (2015) 855-943. (Preprint arXiv: 1211.4230 [math.KT])

[9] V.G. Drinfel'd, On quasitriangular quasi-Hopf algebras and on a group that is closely connected with $\operatorname{Gal}(\overline{\mathbb{Q}} / \mathbb{Q})$, Algebra i Analiz 2:4 (1990) 149-181 (in Russian); Eng. transl. in: Leningrad Math. J. 2:4 (1990) 829-860.

[10] M. Kontsevich, Feynman diagrams and low-dimensional topology, First Europ. Congr. of Math. 2 (Paris, 1992), Progr. Math. 120 (Birkhäuser, Basel, 1994) 97-121.

[11] M. Kontsevich, Homological algebra of mirror symmetry, Proc. Intern. Congr. Math. 1 (Zürich, 1994), (Birkhäuser, Basel, 1995) 120-139. 
[12] M. Kontsevich, Formality conjecture. Deformation theory and symplectic geometry (Ascona 1996, D. Sternheimer, J. Rawnsley and S. Gutt, eds), Math. Phys. Stud. 20 (Kluwer Acad. Publ., Dordrecht, 1997) 139-156.

[13] M. Kontsevich, Derived Grothendieck-Teichmüller group and graph complexes [after T. Willwacher], Séminaire Bourbaki (69ème année, Janvier 2017), no. 1126 (2017) 26 p.

[14] A. Khoroshkin, T. Willwacher and M. Živković, Differentials on graph complexes, Adv. Math. 307 (2017) 1184-1214. (Preprint arXiv: 1411.2369 [q-alg])

[15] C.A. Rossi and T. Willwacher, P. Etingof's conjecture about Drinfeld associators, Preprint arXiv: 1404.2047 [q-alg] (2014) 47 p.

[16] T. Willwacher, M. Kontsevich's graph complex and the Grothendieck-Teichmüller Lie algebra, Invent. Math. 200:3 (2015) 671-760. (Preprint arXiv: 1009.1654 [q-alg])

[17] T. Willwacher and M. Živković, Multiple edges in M. Kontsevich's graph complexes and computations of the dimensions and Euler characteristics, Adv. Math. 272 (2015) 553-578. (Preprint arXiv: 1401.4974 [q-alg])

\section{Appendix A. The heptagon-wheel cocycle $\boldsymbol{\gamma}_{7}$}

In each term, the ordering of edges is lexicographic (cf. Table 1).
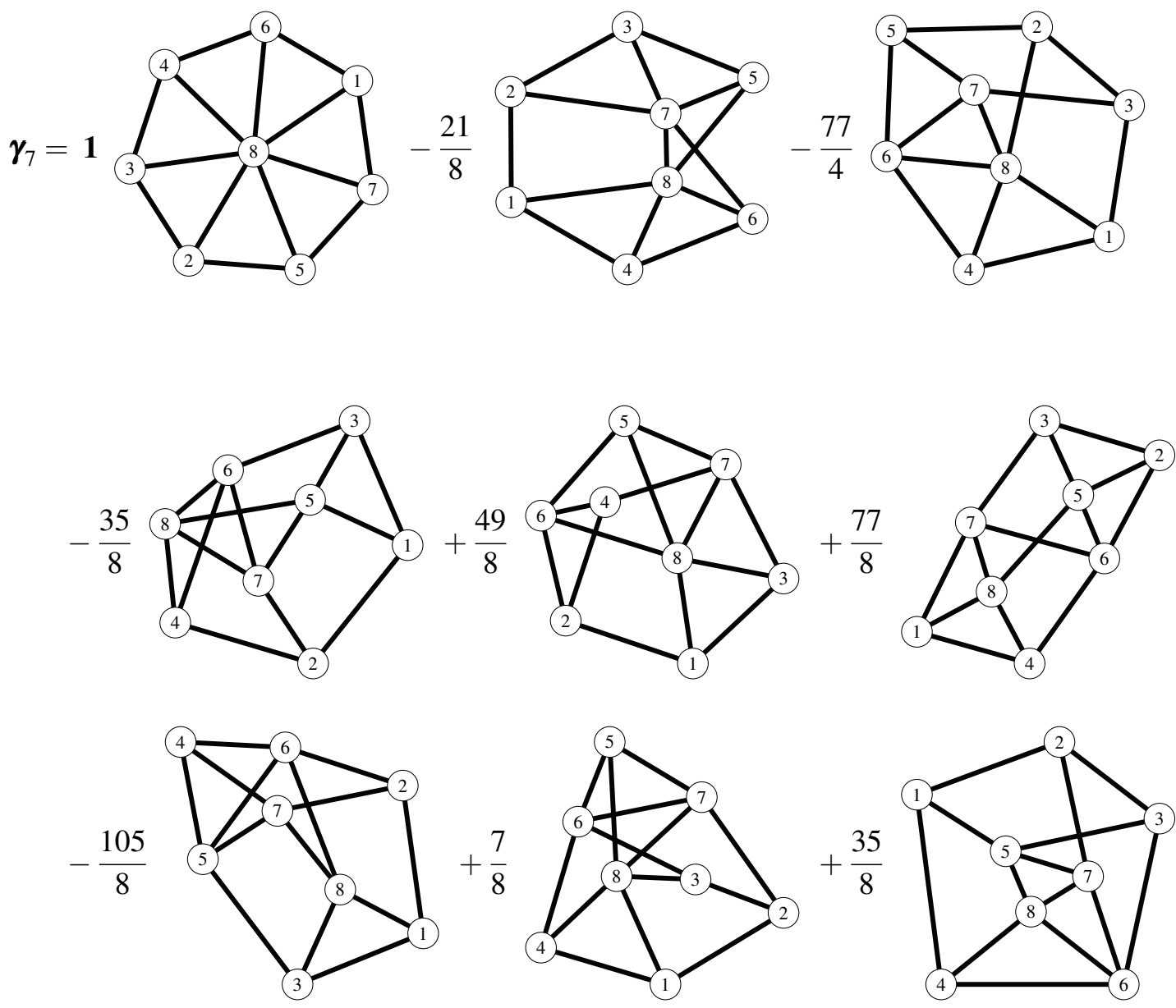

Co-published by Atlantis Press and Taylor \& Francis

Copyright: the authors 

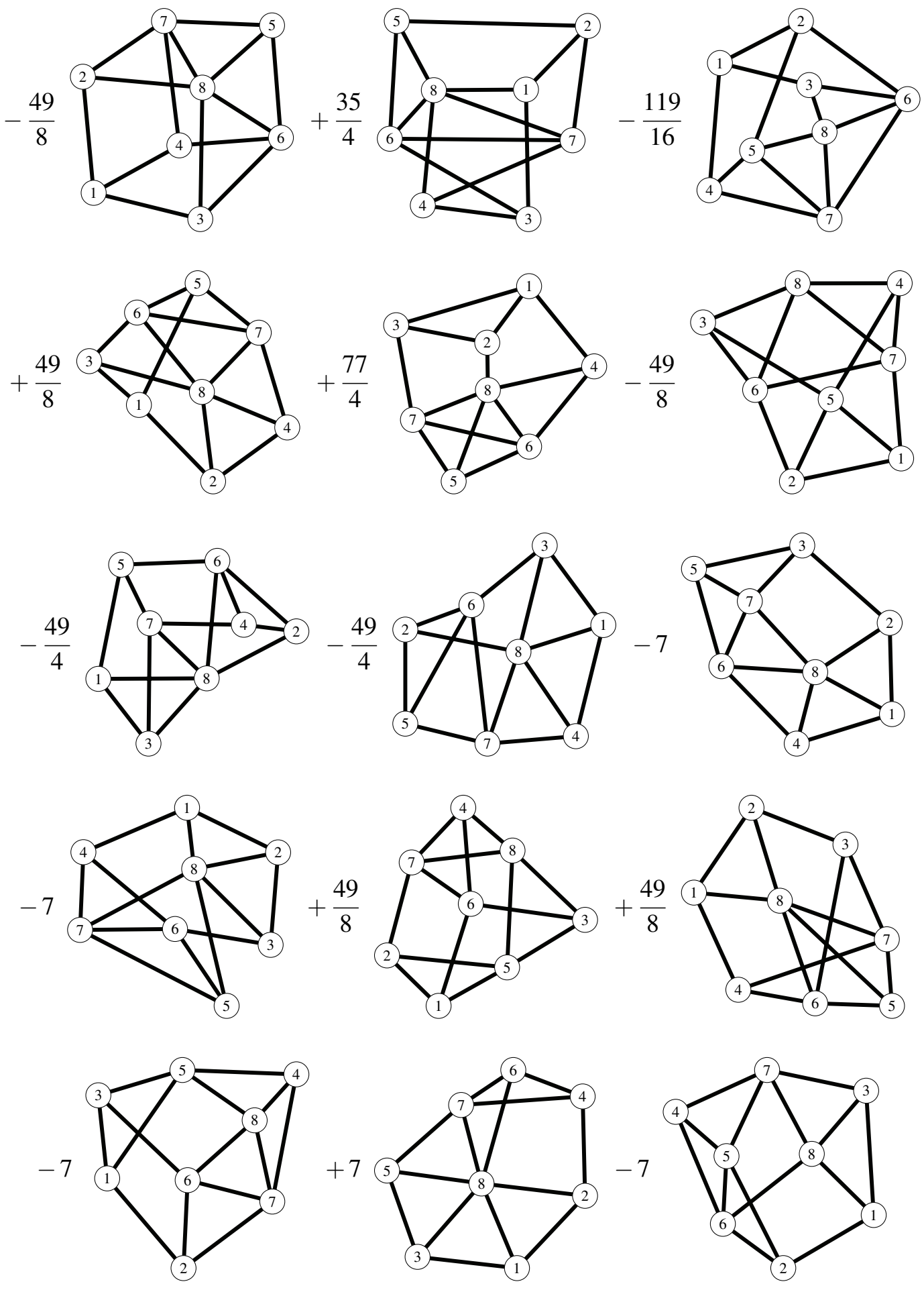

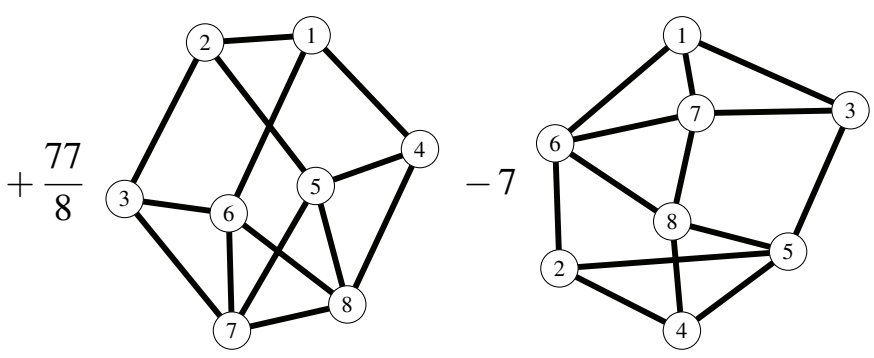

$+\frac{49}{4}$
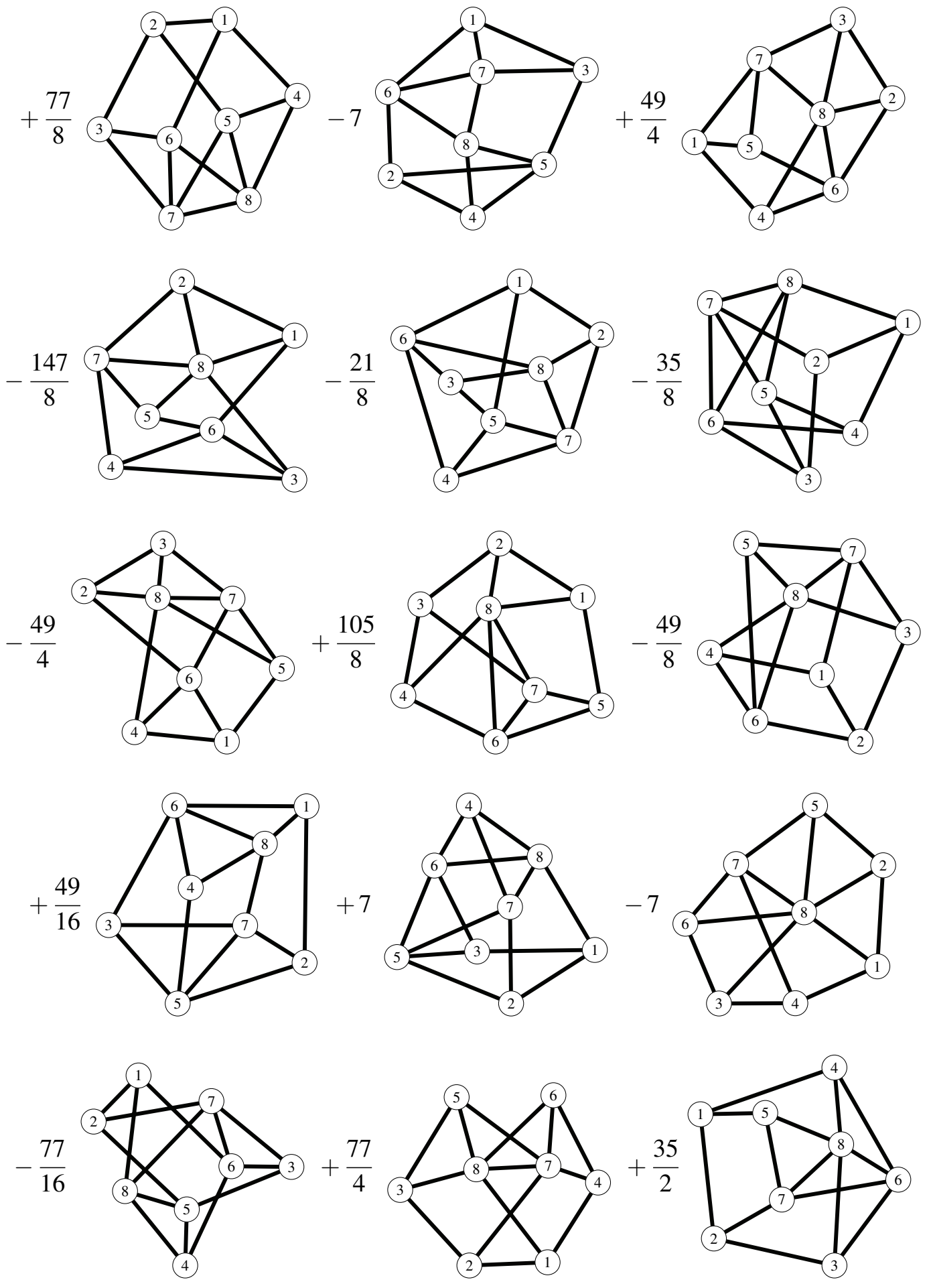

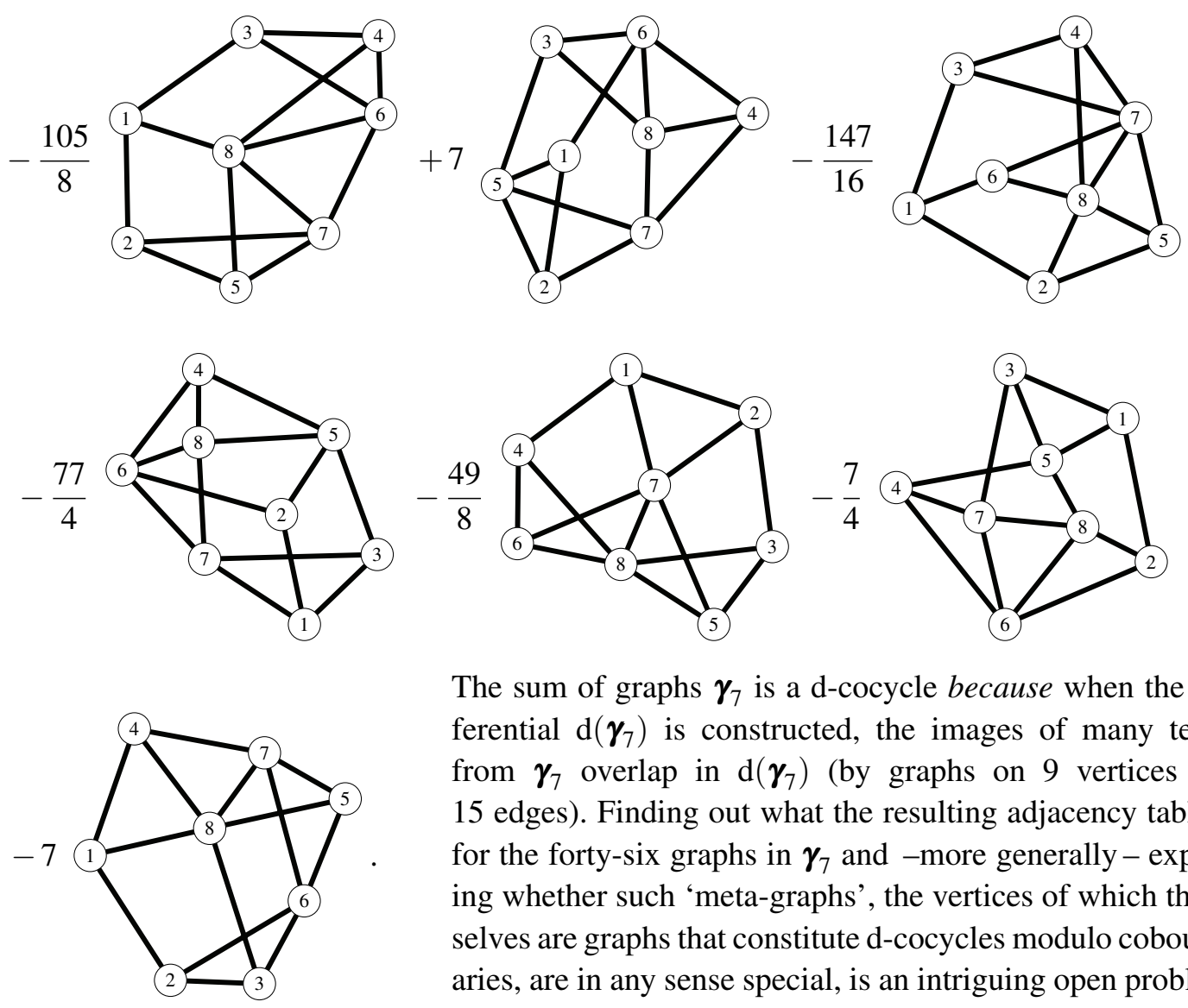

The sum of graphs $\boldsymbol{\gamma}_{7}$ is a d-cocycle because when the differential $\mathrm{d}\left(\boldsymbol{\gamma}_{7}\right)$ is constructed, the images of many terms from $\boldsymbol{\gamma}_{7}$ overlap in $\mathrm{d}\left(\boldsymbol{\gamma}_{7}\right)$ (by graphs on 9 vertices and 15 edges). Finding out what the resulting adjacency table is for the forty-six graphs in $\boldsymbol{\gamma}_{7}$ and -more generally - exploring whether such 'meta-graphs', the vertices of which themselves are graphs that constitute d-cocycles modulo coboundaries, are in any sense special, is an intriguing open problem. (We claim that for $\gamma_{7}$, its meta-graph is connected.)

\section{Appendix B. SAGE code for the graph differential}

The following script, written in SAGE version 7.2, can calculate the differential of an arbitrary sum of non-oriented graphs with a specified ordering on the set of edges for every term, and reduce sums of graphs modulo vertex and edge labelling. ${ }^{j}$ As an illustration, it is shown how this can be used to find cocycles in the graph complex.

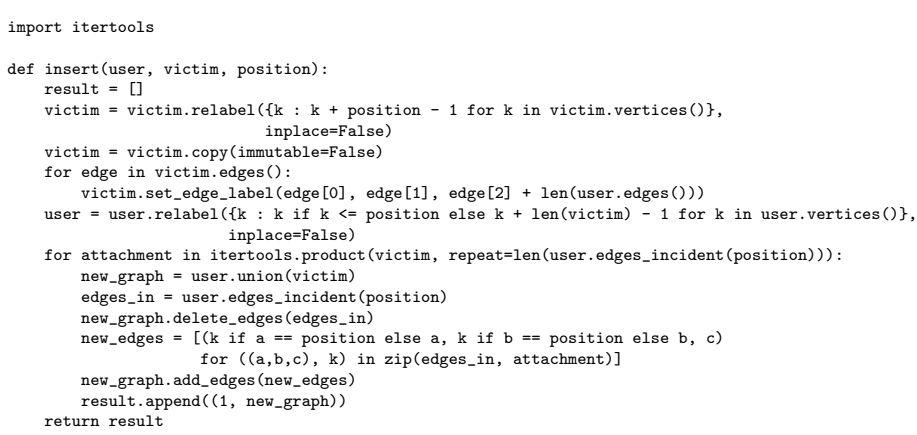

${ }_{\mathrm{j}}$ Another software package for numeric computation of the graph complex cohomology groups in various degrees and loop orders is available from https://github.com/wilthoma/GHoL. 


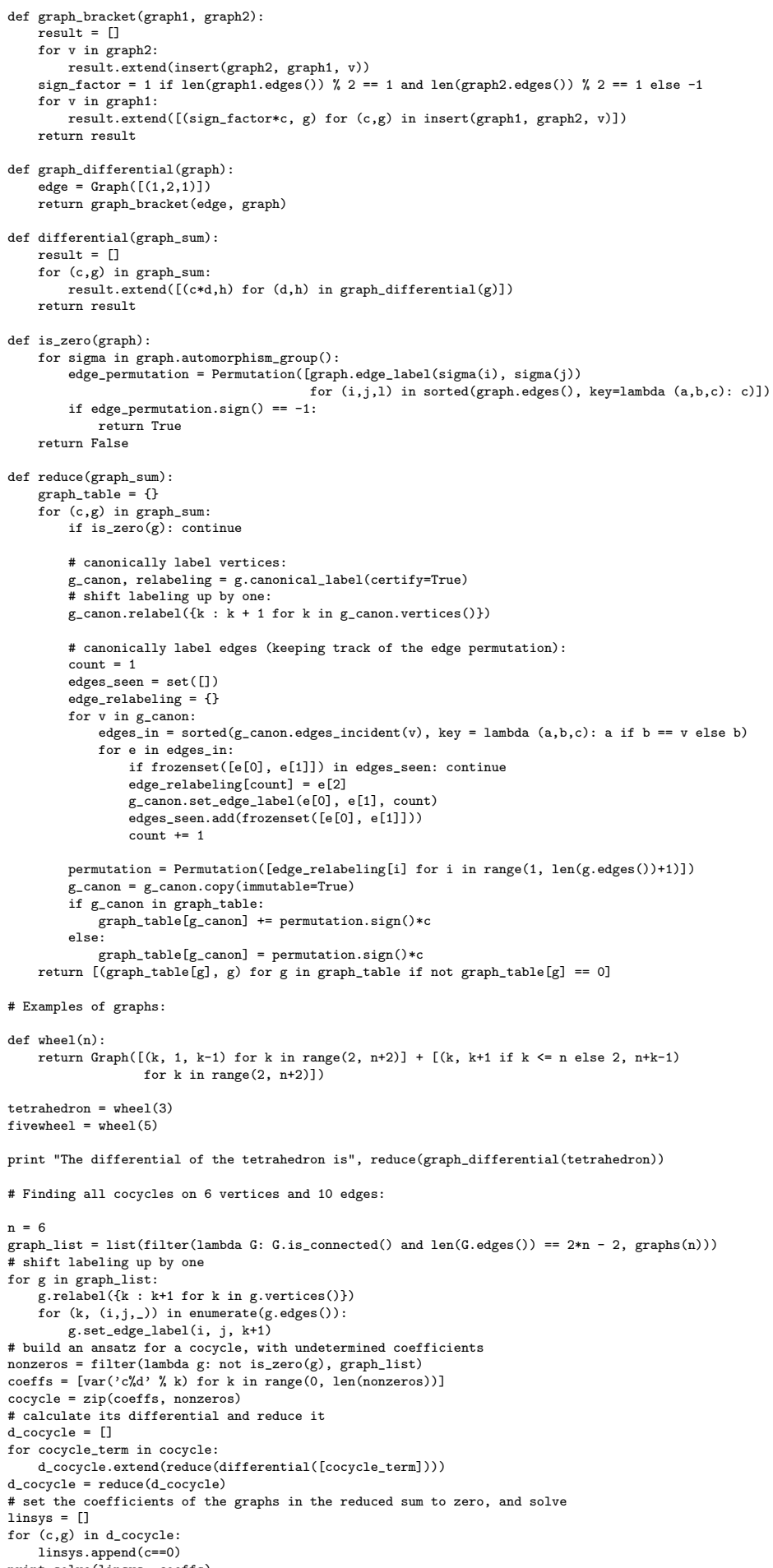

We finally recall that, to the best of our knowledge, the routines by McKay [1] for graph automorphism computation are now used in SAGE (hence by the above program). 